\title{
A Biological Theory of Social Discounting
}

\author{
By Arthur J. Robson and Balázs Szentes*
}

We consider a growth model in which intergenerational transfers are made via stocks of private and public capital. Private capital is the outcome of individuals' private savings while decisions regarding public capital are made collectively. We hypothesize that private saving choices evolve through individual selection while public saving decisions are the result of group selection. The main result of the paper is that the equilibrium rate of return to private capital is at least 2-3\% more than the rate of return to public capital. In other words, social choices involving intertemporal trade-offs exhibit much more patience than individual choices do.

Policy issues often involve intergenerational trade-offs. One highly publicized and polarizing example concerns the appropriate response to global warming. While Nordhaus (1994) concluded that there was no need to enact draconian policies to reduce $\mathrm{CO}_{2}$ emissions immediately, Stern (2006) insisted that the situation was in fact dire. The difference in position can be attributed almost exclusively to the rates of time preference assumed by these two authors-Nordhaus used a few percent, based on estimates deriving from private economic behavior, while Stern essentially set it equal to zero, on the basis of a philosophical stance. It then becomes clear that $\mathrm{CO}_{2}$ emissions should be taken seriously if for some reason private rates of time preference are too high when applied to public decisions, since such decisions should then be made on the basis of a lower rate. ${ }^{1}$

To elaborate further, consider the following hypothetical problem. A policy maker must decide whether to invest in an alternative energy source, which yields some profile of returns for future generations. The internal rate of return of the project is $R$ while the rate of return to private capital is $r$. The positive view of Nordhaus (1994) is based on the idea that implemented policies must reflect the preferences of the current generations. Since, by revealed preference, the current generation is willing to save at rate of return $r$, any project will be implemented as long as $R \geq r$. But what if $R<r$ ? The Pareto criterion offers little guidance in this case. Depending on whether the policy maker discounts the welfare of

* Robson: Department of Economics, Simon Fraser University, 8888 University Drive, Burnaby, British Columbia, Canada V5A 1S6, robson@sfu.ca. Szentes: Department of Economics, London School of Economics, Houghton Street, London, United Kingdom WC2A 2AE, b.szentes@lse.ac.uk. Robson acknowledges support from the Guggenheim Foundation, the Canada Research Chairs program, SSHRC-SFU Small Grants Program, SSHRC Insight Grants program, and the Human Evolutionary Studies Program at SFU. He also thanks participants in the 2013 Toulouse Biology and Economics Workshop and Leanna Mitchell for helpful comments. Szentes thanks the European Research Council for support. The authors declare that they have no relevant or material financial interests that relate to the research described in the paper.

${ }^{1}$ See also Nordhaus (2007). 
the next generation more or less heavily than does the current generation, he might find it desirable either to implement or to abandon it. Stern (2006) argues that an ethical policy maker should discount the next generation less than the current generation and that positive discounting is only justified by the potential extinction of the human race. ${ }^{2}$ Since the probability of this event is deemed to be small, Stern (2006) suggests that the project should be implemented even if $R \approx 0$.

The goal of our paper is to provide a positive theory of private and social discounting and to demonstrate that, in our setting, these discount rates will evolve to be different. We consider an evolutionary model in which members of a society face both private and collective decision problems. Our theory is positive: both of these decisions reflect the preferences of individuals. We show that a discrepancy emerges between the discount factor of an individual applied to private decisions and the one applied to collective decisions. Indeed, individuals exhibit more patience if decisions are made collectively. In the example above, a project might be implemented even if

$$
1+R \leq(1+r) / 2,
$$

where these rates are per generation. We show, by rewriting this inequality in terms of annual rates, that the rate of return on public investements is at least $2-3 \%$ less than the annual rate of return on private capital. This main result implies that, unlike Nordhaus (1994) suggests, the procedure of using data on individual consumption-saving behavior to implement social policies may be seriously flawed, even from a positive point of view. Individuals might welcome the implementation of social projects with significantly lower rates of return than they demand from private projects. However, unlike in Stern (2006), the social discount rate predicted by our theory may be larger than zero and is not pinned down by the probability of extinction of the human race.

Our specific model involves two different intertemporal trade-offs. One trade-off is faced by the individual, while the other is decided collectively. These trade-offs are represented by two different capital stocks in the production function: one private and another public. ${ }^{3}$ Each individual chooses the amount he invests in private capital, but the contribution to public capital is identical for everyone and is selected collectively. Reproduction is sexual, and the return from saving is shared equally among offspring. If a couple has more combined savings, their children each receive more resources in the future, but the couple is left with fewer resources to consume at present, and can only support a smaller family. In other words, consumption-saving decisions involve a quantity-quality trade-off in terms

\footnotetext{
${ }^{2}$ The debate over whether there is an ethical justification for discounting future generations can be traced back as far as Sidgwick (1874), Pigou (1912) and Ramsey (1928).

${ }^{3}$ Using two different types of capital to model private and collective decisions is standard in macroeconomics. For example, the aggregate production technology in Alesina and Rodrik (1994) is identical to ours.
} 
of offspring.

We seek to characterize the saving preferences that arise from an evolutionary process. We hypothesize that individual choices evolve through individual selection, while collective decisions evolve through group selection. More specifically, private saving preferences must be evolutionarily stable, that is, they must be resistant to invasion by rare mutants. Collective decisions must generate the largest possible population growth rate subject to the requirement that private saving decisions are evolutionarily stable.

Our main finding is that individuals are significantly more patient with regard to collective decisions than private ones. As a consequence, the equilibrium gross return to public capital is less than half the gross return to private capital over the period of one generation. This result is solely due to the sexual nature of reproduction. Because two parents are required for reproduction, private incentives to invest in children are cut in half. In biological terms, individual selection forces private saving decisions to obey Hamilton's rule. According to this rule, individuals behave as if children were half as valuable as they themselves are, with grandchildren worth only one quarter as much. This implies that private saving decisions exhibit too much impatience relative to biologically efficient behaviour.

To develop an intuition for our main result, note that the equilibrium private saving behavior must be resistant to rare mutants. The most threatening rare mutants are those that maximize their growth rate by taking into account the fact that they are rare in the population. Mutants have the option to mimic the saving behavior of the original population, so rare mutants can always do at least as well. Hence, the only way to ensure that mutants cannot invade is for the saving behavior of the original population to match that of the most threatening mutants. That is, the general population must maximize their growth rate essentially as if their associated gene had zero frequency. ${ }^{4}$ In the context of sexual reproduction, if a gene has zero frequency, then its carriers are only half-related to their children and only one-quarter-related to their grandchildren. For this reason, individuals behave as if children were twice as valuable as grandchildren.

This behavior distorts the quality-quantity trade-off involving offspring when compared with asexual reproduction. Indeed, if reproduction were asexual, children and grandchildren would be of equal value. Therefore, the private saving decision which maximizes fitness would equate the marginal return to saving measured in the number of grandchildren with the marginal return to consumption in terms of offspring. This saving decision turns out to be the one that maximizes population growth, resulting in biological efficiency. When reproduction is sexual, individuals prefer to sacrifice some grandchildren by increasing their number of children at the cost of investing less in each child. Therefore, relative

\footnotetext{
${ }^{4}$ Bergstrom (1995) analyzes games played by siblings. He concludes that the strategy that cannot be invaded by a mutant involves choosing an action as if the opponent mimics this action with probability one-half. The same principle is at work here. That is, although in equilibrium siblings have the same genes and take the same actions, they behave as if this probability were only one-half, which is what it would be if their type were rare.
} 
to the asexual case, couples have a preference for too many children, each of lower quality. Equivalently, private investment is made too impatiently. This induces a slower growth rate and fewer children in the equilibrium steady state. While the public saving decision is technologically analogous to the private investment, this decision is made collectively and must be followed by each member of the community. It evolves through group selection to maximize population growth, with resulting saving behavior that is similar to the behavior that emerges in the asexual case. Therefore, this collective saving choice is guided by a lower rate of time preference. Indeed, the public decision may involve still greater investment to partially, but not completely, offset the distortion created by insufficient private investment.

We intend for our model to be relevant in a variety of contexts. For example, it could be applied to the exhaustion of a depletable resource, or pollution of the local environment. We introduce a specific growth model with two capital stocks and a steady state merely to facilitate the analysis. Our basic qualitative conclusions seem bound to hold in many models involving intertemporal tradeoffs.

\section{Related Literature}

There is a small but steadily growing literature investigating the relationship between biology and economic behavior - see, for example, Robson and Samuelson (2010) and the references therein. Further, some of these papers examine how time preferences evolved through individual selection - see, for example, Rogers (1994), Robson and Szentes (2008), and Robson, Szentes and Iantchev (2012). The basic conclusion of these papers is that the evolutionarily stable choice behavior can be represented by time-separable utility. The discount rate is the sum of the mortality rate and the population growth rate, and the overall utility function is reproductive value. Robson and Samuelson (2009) shows that this conclusion crucially depends on the assumption that all the risk is idiosyncratic. They argue that if there is aggregate uncertainty the evolutionary stable behavior no longer has an expected utility representation. In all these papers, all the decisions are made at the individual level. Our primary goal here, in contrast, is to highlight a discrepancy between preferences for individual and collective choices.

There are also a few papers that consider macroeconomic phenomena in a biological context. These involve standard growth models with the twist that the reproduction technology is specified instead of the preferences of individuals. The goal then is to characterize the choices that induce the largest population growth rate. Hansson and Stuart (1990) consider a neoclassical growth model in which clans compete for shares of resources. A clan's production depends on the stock of clan-specific capital and on the total population of all clans. The clans indeed face a carrying capacity constraint, meaning that per capita production decreases as total population rises. An individual is active for only one period, and each clan faces a consumption-saving decision. Higher consumption results in higher immediate population growth but a smaller capital stock, and hence smaller out- 
put, in the next generation. The carrying capacity constraint means that each clan's size must be constant in the long-run equilibrium. As a result, evolution selects clans with a zero rate of time preference. Agents maximize the per capita steady state consumption of current and future generations.

Robson and Wooders (1997) also consider a macroeconomic model where total output depends on different types of capital and labor. The per capita income of each type of labor determines its growth rate. The authors show that when the balanced growth rate is maximized, income must be distributed across individuals in accordance with marginal product pricing. In both Hansson and Stuart (1990) and Robson and Wooders (1997), reproduction is asexual and all the choices are made collectively. In other words, these models are similar to our model without private capital.

\section{The Model}

Time is discrete and there is a continuum of individuals. ${ }^{5}$ In each period, adult individuals are randomly matched, allocate resources to reproduction and capital investment, and die. The newborns become the adults of the next period, and the return on investment is then available. We next describe the technologies for production and reproduction.

There is a single aggregate output which is produced from public capital, $M$, private capital, $K$, and labor, $L$. The aggregate output available at time $t+1$ is given by the function $G\left(M_{t}, K_{t}, L_{t}\right)$ where $M_{t}, K_{t}$ and $L_{t}$ are the levels at time $t$ of public capital, private capital, and labour, respectively. (More precisely, $L_{t}$ is the number of adults at time $t$.) The depreciation rates of public and private capital are $\delta_{M}$ and $\delta_{K}$, respectively.

We assume the production function $G$ exhibits constant return-to-scale. Defining $m=M / L$ and $k=K / L$ as per capita public and private capital, respectively, we then have

$$
\begin{aligned}
\frac{G(M, K, L)+\left(1-\delta_{M}\right) M+\left(1-\delta_{K}\right) K}{L} & =G(m, k, 1)+\left(1-\delta_{M}\right) m+\left(1-\delta_{K}\right) k \\
& =g(m, k),
\end{aligned}
$$

say, where $g(m, k)$ is the gross per capita output. That is, incorporating depreciation of this linear form is merely a matter of redefining the production function. We assume that the function $g$ is three times continuously differentiable, satisfies Inada conditions in each input, is strictly concave, and has the inputs as (weak) complements. ${ }^{6}$

The number of offspring a couple has depends on their total consumption,

\footnotetext{
${ }^{5}$ Presumably, one half of the individuals are male and one half female. There is, however, no need for a formal distinction in the model between the sexes.

${ }^{6}$ That is, more precisely:

i) $g(0, k)=g(m, 0)=0$, for all $k, m \geq 0$,

ii) $g_{m}(m, k)>0, g_{k}(m, k)>0$, for all $k, m>0$,
} 
where consumption is the residual resources left after investment. Suppose that one individual in a couple has resources $w_{1}$ and privately saves $k_{1}$, whereas the other has resources $w_{2}$ and saves $k_{2}$. Then, if the per-capita public capital is fixed at $m$, they consume $c_{1}=w-k_{1}-m$ and $c_{2}=w-k_{2}-m$, respectively. The expected number of offspring of a couple then depends on $c_{1}$ and $c_{2}$, and is given by $2 f\left(c_{1}, c_{2}\right) .{ }^{7}$ The reproduction function $f$ is symmetric, twice jointly continuously differentiable, $f(0,0)=0$, and $f_{1}, f_{2}>0$, for all $c_{1}, c_{2} \geq 0$, where $f_{1}$ and $f_{2}$ denote the partial derivatives of $f$. We assume that each individual has access to the production technology $g$. Hence, if the parents invest $k_{1}$ and $k_{2}$ in private capital and the per-capita public capital is $m$ then each of their offspring receive resources

$$
\frac{g\left(m, k_{1}\right)+g\left(m, k_{2}\right)}{2 f\left(c_{1}, c_{2}\right)} 8
$$

We assume that choices regarding private saving evolve through individual selection while the decisions regarding public capital are shaped by group selection. Consider many isolated communities, each of which is characterized by a percapita level of public capital $m$. Private saving behavior is genetic; each offspring inherits the choice behavior of one of her parents with probability half. Private savings behavior is the result of individual selection if it is resistant to invasion by any mutant, so that no mutant can grow faster than the original community. Such behavior is an evolutionarily stable strategy, ESS, and we show that, for each $m$, there is a unique ESS denoted by $\bar{k}(m)$. Finally, a per-capita level of public capital $\bar{m}$ is the result of group selection if the community characterized by $\bar{m}$ grows faster than any other community subject to the constraint that the private saving is an ESS in every community. More formally, we will identify the per-capita public capital $\bar{m}$ which is feasible and maximizes the population growth subject to the constraint that the private saving is given, as a function of $m$, by $\bar{k}(m)$.

Group Selection as Equilibrium Selection-Our assumption that collective decisions are driven by group selection merits justification. This concept is that competition does not only take place at the level of individuals but also at the

$$
\begin{aligned}
& \text { iii) } g_{m}(m, k) \rightarrow\left\{\begin{array} { c } 
{ \infty , \text { as } m \rightarrow 0 , \text { for all } k > 0 } \\
{ 0 , \text { as } m \rightarrow \infty , \text { for all } k \geq 0 }
\end{array} \text { and } g _ { k } ( m , k ) \rightarrow \left\{\begin{array}{c}
\infty, \text { as } k \rightarrow 0, \text { for all } m>0 \\
0, \text { as } k \rightarrow \infty, \text { for all } m \geq 0
\end{array}\right.\right. \text {, } \\
& \text { iv) } g_{m m}(m, k)<0, g_{k k}(m, k)<0 \text { and } g_{m m}(m, k) g_{k k}(m, k)-\left(g_{m k}(m, k)\right)^{2}>0 \text { for all } m, k>0, \\
& \text { v) } g_{m k}(m, k) \geq 0 \text { for all } m, k>0 \text {. }
\end{aligned}
$$

\footnotetext{
${ }^{7}$ This formulation implicitly allows for mortality. That is, if the expected number of newborn offspring per couple is $2 \bar{f}\left(c_{1}, c_{2}\right)$, and a fraction $S$ of these survive to adulthood, then we can simply set $f\left(c_{1}, c_{2}\right)=$ $S \bar{f}\left(c_{1}, c_{2}\right)$.

${ }^{8}$ Alternatively, we could have assumed that individuals can save in a competitive market for capital. That is, if an individual saves $k^{\prime}$ and the population average of private capital is $k$ then his offspring receive $k^{\prime} g_{k}(m, k)$ resources. Such an assumption has no impact on our results but seems less plausible in an evolutionary model.
} 
group level. It posits that a particular choice behavior is more likely to survive evolution if a group whose members practice this behavior grows faster than other groups. Group selection merits skepticism in instances where individuals sacrifice their individual fitness to aid the group; see, for example, Williams (1972) and Maynard Smith (1964). Group selection is less controversial and is widely accepted in situations where it is not opposed by the force of individual selection. Group members in such situations typically face a coordination problem; e.g. adaptation of a social structure, or formation of a social norm, see Boyd and Richerson (1990). In our model, the only individual choice is the private capital investment and it is determined solely by individual selection. For example, one salient form of such private investment that would be available even to huntergatherers would be somatic investment in the size of each child. ${ }^{9}$ Group selection is only employed to pin down the level of public capital. We assume that the technology of public capital dictates that the level of public investment must be the same for each group member, irrespective of whether or not he is a mutant. For example, one form of such public investment might be a plan to migrate as a group to to a different region. ${ }^{10}$ Thus our appeal to group selection is limited and therefore plausible in the light of evolutionary biology.

\section{Results}

We first characterize the biologically efficient steady state saving decisions. That is, we identify the public and private capital levels which are feasible and which maximize the growth rate of the population. This serves as a benchmark and allows us to identify the biological inefficiency due to individual selection.

Suppose that $k, m$ and $c$ are constant over time. ${ }^{11}$ For feasibility, the triple $(m, k, c)$ has to satisfy the budget constraint that

$$
\frac{g(m, k)}{f(c, c)}=m+k+c
$$

where the left-hand-side is the inheritance of an offspring from her parents, as in (2), and the right-hand-side is the allocation of these resources to investments and consumption. Lemma 1 of the Appendix shows that each pair $m, k \geq 0$ uniquely determines the feasible level of consumption, $c(m, k) \geq 0$, say. If each couple has $2 f(c, c)$ offspring, the growth factor of this population is $f(c, c)$. Since $f$ is strictly increasing, the problem of maximizing the growth factor of the population

\footnotetext{
${ }^{9}$ Taking this interpretation seriously would suggest some slight changes in the model specification but these would not change the qualitative results.

${ }^{10}$ Slightly more generally, individuals might be able to choose not to migrate with the group, where such a choice bears a prohibitively high cost.

${ }^{11}$ All the results here are based on such a steady state. However, it seems that similar qualitative results could be derived from a full dynamic trajectory. In particular, a decrease in concern for offspring due to sexual reproduction would arise out of the steady state.
} 
is that of

$$
\max _{m, k \geq 0} c(m, k)
$$

subject to the budget constraint (3). To develop intuition, suppose that the solution is interior and the first-order approach is valid. ${ }^{12}$ Then, replacing $c$ by $c(m, k)$ in Equation $(3)$ and differentiating both sides by $m$ yields

$$
\left(f_{1}(c, c)+f_{2}(c, c)\right) c_{m}(m, k)(c+m+k)+f(c, c)\left(c_{m}(m, k)+1\right)=g_{m}(m, k) .
$$

If $(m, k)$ induces the largest possible consumption then $c_{m}=0$, so the previous equation simplifies to $f(c, c)=g_{m}(m, k)$. An analogous argument shows that $f(c, c)=g_{k}(m, k)$. We have:

THEOREM 1: There is a unique pair $m, k>0$ which induces the largest feasible consumption $c>0{ }^{13}$ This pair is characterized by the first-order conditions

$$
g_{m}(m, k)=g_{k}(m, k)=f(c, c) .
$$

\section{PROOF:}

See the Appendix for a rigorous proof.

These optimality conditions equate the marginal product of each type of capital to the growth factor of population, $f(c, c)$. A key "wrinkle" is that the growth factor of population is endogenous, indeed it is the objective to be maximized.

The above formulation is in discrete time, where each period represents a generation. This can be translated into a more familiar annual formulation as follows. Suppose the intergenerational time is $T$ years, say. Consider the annual rates of return on public and private capital are $r_{m}$ and $r_{k}$, say, where these are compounded continuously, for simplicity. Similarly suppose the growth rate of population is $\pi$ per annum, where this is also compounded continuously. The first order conditions are then $g_{m}(m, k)=e^{r_{m} T}=g_{k}(m, k)=e^{r_{k} T}=f(c, c)=e^{\pi T}$ which reduce to $r_{m}=r_{k}=\pi$.

We take the implied pure rate of time preference, $\rho$, say, as the value that would support these choices if preferences were given in the conventional simple intertemporal form and there were no growth in consumption. We have then $\rho=r_{m}=r_{k}=\pi$.

We turn now to formulating the problem with sexual reproduction. For each $m$, we compute the unique steady state investment in private capital which is immune to invasion by a rare mutant. Suppose that, in the candidate steady state equilibrium, each non-mutant adult allocates $m$ to investment in public capital, $\bar{k}$ to private capital, leaving $\bar{c}$ to promote population growth. Consider

\footnotetext{
${ }^{12}$ The proof of Theorem 1 in the appendix rules out corner solutions and validates the first-order approach.

${ }^{13}$ We abuse notation by using $m, k$ for the general values of these variables, as well as the specific optimal choices of them.
} 
then a rare mutant that must also allocate $m$ to public capital, but allocates $k$ to private capital, leaving $c$ to promote growth.

Since mutants are rare, essentially all the matches involving mutants have one mutant and one non-mutant. We then calculate the maximum steady state growth factor for the mutants in this situation, so the mutant attains such steady state growth while remaining rare. The growth factor of the mutant population is $f(\bar{c}, c)$. That is, each mutant-non-mutant couple has $2 f(\bar{c}, c)$ offspring, one half of whom are mutants, on average. By (2), the budget constraint of a mutant is

$$
m+k+c=\frac{g(m, \bar{k})+g(m, k)}{2 f(\bar{c}, c)} .
$$

Lemma 2 of the Appendix shows that, although there may exist values of $k$ for which (4) cannot be satisfied for any $c \geq 0$, there must be some values for which it can be satisfied. (If it cannot be satisfied, even $c=0$ will not permit the attainment of a steady state.) If (4) can be satisfied, Lemma 2 shows it has a unique solution, $c(k)$, say, which is twice differentiable. The problem of maximizing the growth rate of the mutants is then:

$$
\begin{gathered}
\max c(k) \\
\text { over } k \in \mathbb{R}_{+} \text {such that (4) is satisfied. }
\end{gathered}
$$

Lemma 2 also shows that, when (4) can be satisfied, there is a unique solution for $c$ given by $c(k)$. Proceeding informally, then, it follows from (4) that

$$
2 f_{2}(\bar{c}, c(k)) c^{\prime}(k)(m+k+c(k))+2 f(\bar{c}, c(k))\left(1+c^{\prime}(k)\right)=g_{k}(m, k) .
$$

If $k$ generates the largest possible level of consumption, then $c^{\prime}(k)=0$, so the previous equation becomes

$$
2 f(\bar{c}, c(k))=g_{k}(m, k) .
$$

To complete the informal argument, note that mutants match the growth rate of the original population if they invest $\bar{k}$ in private capital, but they maximize their growth rate by choosing $k$. So, unless $k=\bar{k}$, the mutants can invade the original population. In short, $(\bar{k}, \bar{c})$ is an evolutionarily stable strategy, ESS, if and only if $k=\bar{k}$ and $c=\bar{c}$. We have:

THEOREM 2: For each $m>0$, there exists a unique (pure strategy) ESS $(\bar{k}, \bar{c})$ which satisfies

$$
g_{k}(m, \bar{k})=2 f(\bar{c}, \bar{c}) \cdot{ }^{14}
$$

\section{PROOF:}

\footnotetext{
${ }^{14}$ We again abuse notation slightly by using $\bar{m}, \bar{k}$ to denote the values of these variables that might arise in the general population, as well as the specific ESS values of them.
} 
See the Appendix for a rigorous proof.

The equilibrium condition determining $\bar{k}$ equates the marginal product of private capital to twice the population growth factor. Other things equal, then, the equilibrium choice of private capital would be much too low.

The effect of sexual reproduction is to substantially increase impatience and therefore reduce investment in private capital. The mutants try then to have a larger number of offspring by reducing the income these offspring will have in the next period. The cost of this would be a reduced potential number of grandchildren. ${ }^{15}$ However, in the steady state, since income falls, the ultimate effect of the mutant is to reduce the number of children and the growth factor.

In a conceptual sense then the mutant is trading off immediate rewards (children) against a delayed reward (grandchildren) and leaning too much in favor of the immediate reward. Sex then in this model is an underlying cause of too much impatience.

Consider now how the optimal level of public capital, $\bar{m}$, say, is determined by group selection. ${ }^{16}$ Not only should the group choose on the basis of a lower and more appropriate rate of time discount, but the group should increase $m$ still further in order to offset the deleterious effect of too low a value of $k$. The optimal public capital maximizes the growth rate subject to the feasibility constraint and to private saving being determined as above by individual selection. Formally, $\bar{m}$ is the solution of the problem ${ }^{17}$

$$
\text { subject to } f(c, c)(m+k+c)=\quad \begin{gathered}
\max _{m} c \\
g(m, k) \text { and } g_{k}(m, k)=2 f(c, c),
\end{gathered}
$$

where the first constraint is the feasibility constraint, and the second constraint ensures that the private saving decision is an ESS (see Theorem 2). Again, we content ourselves here with an intuitive characterization of the solution, leaving a rigorous treatment to the Appendix. Suppose $\bar{c}(m)$ and $\bar{k}(m)$ satisfy the constraints. Differentiate both sides of the first constraint with respect to $m$ to

\footnotetext{
${ }^{15}$ More precisely, what is reduced is the income available to children. This income can be used to produce new children, but also for investment to generate the income of the new children when they, in turn, become adults.

${ }^{16}$ As noted already, the full force of group selection is not needed if the values of $m$ are locked together. For example, perhaps the issue whether to pay the cost of a long trek across barren land to a land more fertile than that occupied at present. However, either the entire group will do this or no-one will. Any plausible political scheme will then generate the optimal choice. This is akin to "solving" the prisoner's dilemma by restricting attention to the diagonal.

${ }^{17}$ For clarity, we spell out both relevant constraints, and avoid notation derived from satisfying one or both of them.
} 
get

$$
\begin{aligned}
& {\left[f_{1}(\bar{c}(m), \bar{c}(m))+f_{2}(\bar{c}(m), \bar{c}(m))\right] \bar{c}^{\prime}(m)(m+\bar{k}(m)+\bar{c}(m)) } \\
& +f(\bar{c}(m), \bar{c}(m))\left(1+\bar{k}^{\prime}(m)+\bar{c}^{\prime}(m)\right) \\
= & g_{m}(m, \bar{k}(m))+g_{k}(m, \bar{k}(m)) \bar{k}^{\prime}(m) .
\end{aligned}
$$

If $\bar{m}$ maximizes $\bar{c}$ then $\bar{c}^{\prime}(\bar{m})=0$. Using the second constraint as well, we then have

$$
f(\bar{c}(\bar{m}), \bar{c}(\bar{m}))\left(1-\bar{k}^{\prime}(\bar{m})\right)=g_{m}(\bar{m}, \bar{k}(\bar{m})) .
$$

It follows that $\bar{k}^{\prime}(\bar{m}) \geq 0$, since the two types of capitals are complements. We have:

THEOREM 3: Group selection for the level of public capital, given individual selection for the level of private capital, generates a level of public capital $\bar{m}>0$, private capital $\bar{k}(\bar{m})>0$ and consumption $\bar{c}(\bar{m})>0$ which satisfy

$$
g_{m}(\bar{m}, \bar{k}(\bar{m}))=f(\bar{c}(\bar{m}), \bar{c}(\bar{m}))\left(1-\bar{k}^{\prime}(\bar{m})\right)<2 f(\bar{c}(\bar{m}), \bar{c}(\bar{m}))=g_{k}(\bar{m}, \bar{k}(\bar{m})) .
$$

In the special case that $g_{m k}(m, k)=0$, it follows that $g_{m}(\bar{m}, \bar{k}(\bar{m}))=f(\bar{c}(\bar{m}), \bar{c}(\bar{m})) \cdot{ }^{18}$

\section{PROOF:}

See the Appendix for a rigorous proof.

That is, the marginal product of public capital is no greater than half the marginal product of private capital. In fact, the marginal product of public capital will be reduced below the population growth factor precisely because the marginal product of private capital is above the population growth factor and increasing public capital generally increases the equilibrium level of private capital.

Again, it is helpful to translate these conditions into annual form. Suppose the continuously compounded annual rates of return on private and public capital are $r_{k}$ and $r_{m}$ respectively, the continuously compounded annual growth rate of population is $\pi$ and the intergenerational period is $T$ years. It follows that $g_{k}=e^{r_{k} T}=2 f=2 e^{\pi T}$ so that $r_{k}=\pi+\ln 2 / T$. In addition, it follows that $g_{m}=e^{r_{m} T} \leq f=e^{\pi T}$ so that $r_{m} \leq \pi$. We take the implied pure rates of time preference on private and social capital, $\rho_{k}$ and $\rho_{m}$, say, respectively, as those that support these choices for conventional preferences and no growth. It follows that $\rho_{k}=r_{k}=\pi+\ln 2 / T$ and that $\rho_{m}=r_{m} \leq \pi$. The difference between the pure rates of time preference $\rho_{k}$ and $\rho_{m}$ is then at least $\ln 2 / T$. Perhaps a low-side estimate for the average intergenerational time for humans is 25 . If $T=25$, then $\ln 2 / T=2.77 \%$. A high-side estimate might be $T=30$, in which case $\ln 2 / T=2.31 \%$. That is, the pure rate of time of time preference for social investment is at least $2-3 \%$ lower than the rate for private investment.

\footnotetext{
${ }^{18}$ If $g_{m k}(m, k)<0$, it follows that $g_{m}(\bar{m}, \bar{k}(\bar{m}))>f(\bar{c}(\bar{m}), \bar{c}(\bar{m}))$. However, unless $m$ and $k$ are very strong substitutes, this would not overturn the conclusion that $g_{m}(\bar{m}, \bar{k}(\bar{m}))<g_{k}(\bar{m}, \bar{k}(\bar{m}))$.
} 
Evidence on Private versus Social Rates of Discount - In the light of the above argument, the following issue is crucial. Wouldn't our analysis predicting that the pure rate of time preference for private investment is higher than the similar rate for public investment fly in the face of the stylized fact that public investment is too low in modern economies? This is a complex issue, and the public rate of time preference is not the only factor involved in the political process determining public investments. The stylized fact could be buttressed by concentrating on particular sectors in particular countries. Perhaps investment in public transit in the US would pay off handsomely in the future, for example.

From the present point of view, however, it seems appropriate to focus on overall rates of return. There is not a great deal of relevant empirical work here. One exception is Afonso and St Aubyn (2010) who use data from the 14 EU countries plus Canada, Japan and the US to estimate the macroeconomic effect of impulses in public and private investment. ${ }^{19}$ What they describe as the total rates of return on public and private investment, seem most relevant here. The partial public rate of return on public investment, for example, attributes all of the extra output consequent on an impulse investment in public capital to that impulse. The total public rate of return, more appropriately, attributes the extra output not only to the impulse in public investment but also to the private investment that this stimulates. Table 1 of their paper summarizes these rates of return. These rates are, in turn, derived from marginal products of investment. They do not calculate the rates if the marginal product is negative, but these cases are the most favorable to our thesis, so we do not omit them. ${ }^{20}$ The total marginal product following an impulse in public investment is less than the total marginal product following an impulse in private investment for 9 of the 17 countries. Furthermore, the simple average of the 17 total marginal products following an impulse in public investment is 1.38 , whereas the simple average of the total marginal products following an impulse in private investment is 1.09 . With the 20 year horizon used, the associated rates of return are $0.44 \%$, and $1.62 \%$, respectively. The difference of $1.18 \%$ is in the direction predicted by the present model, although somewhat less. ${ }^{21}$ It is obvious that more empirical

\footnotetext{
${ }^{19}$ Afonso and St Aubyn claim to be the first to systematically compute and compare public partial and total investment rates of return.

${ }^{20}$ Conceivably, these estimated marginal productivities are somehow uniformly biased downwards.

${ }^{21}$ Consider how these data might arise in the context of the current model. Suppose the levels of public and private capital are detemined by the equilibrium conditions- $g_{m}(m, k)=r_{m}$ and $g_{k}(m, k)=r_{k}$. If public capital increases, it follows that the private capital changes according to $\frac{d k}{d m}=-\frac{g_{k m}}{g_{k k}}>0$, so there is "crowding-in". It also follows that $\frac{d g}{d m}=g_{m}-g_{k} \frac{g_{k m}}{g_{k k}}>r_{m}$, so that the "partial" rates of return indeed overestimate the true rates of return. On the other hand, the "total" rate of return, consequent on an impulse in public investment, as in Afonso and St Aubyn, would be $T R R_{m}=\frac{\frac{d g}{d m}}{1+\frac{d k}{d m}}=\frac{r_{m}-r_{k} \frac{g_{k m}}{g_{k k}}}{1-\frac{g_{k m}}{g_{k k}}}$. Thus $T R R_{m}=r$ in the case that $r_{m}=r_{k}=r$. Although the total rate of return is not generally $r_{m}$ it may be a more reasonable estimate than the partial rate of return. Of course, an analogous expression holds for $T R R_{k}$, the total rate of return consequent on a impulse of private investment. After some algebra, it can be shown that that $T R R_{k}-T R R_{m}=\left(r_{k}-r_{m}\right) \Delta$, where $\Delta=\frac{g_{k k} g_{m m}-g_{k m}^{2}}{\left(g_{k k}-g_{k m}\right)\left(g_{m m}-g_{k m}\right)} \in(0,1]$. That
} 
research is required to satisfactorily address this issue. However, the results so far do not provide much support for the stylized fact, but leave open the contrary possibility that the public rate of time preference is less than the private rate, as in the present paper.

\section{Further Discussion}

Carrying Capacity - In the foregoing, we maximize population growth, with no restriction on what this maximum rate might be. Clearly, however, limited carrying capacity would ultimately force the equilibrium growth factor to be one (as in Hansson and Stuart (1990)). The simplest apparent resolution of this issue would be to build in to the production function a negative feedback effect of total population. If the production function has the maximal population level built in, the above analysis then applies directly, with a growth rate of zero.

The difficulty with this resolution is that, although zero population growth does not affect the gap between the private and social rates of time preference found here, it does affect their absolute levels. This may conflict with other empirical evidence on these rates. A resolution is provided by Robson and Samuelson (2009) who consider a general setting in which mortality rates are subject to aggregate shocks. Consider, for example, a model with periods of tranquil growth, interrupted by the occasional random disaster during which the population is drastically reduced..$^{22}$ Rates of time preference would then be those appropriate to the tranquil periods of steady growth, with the catastrophes merely serving to lower growth to a plausible level. The bottom line is that the present results can be taken as is, with no constraint set by a zero growth rate.

Behavior versus Preferences - The model here is cast directly in terms of behavior rather than indirectly in terms of preferences. The indirect approach to preferences might produce a divergence between preferences and fitness when preferences are treated as a strategic choice, where this choice is revealed to an opponent. In such circumstances, it may pay to set a preference profile that differs from fitness in order to induce an opponent to modify his/her behavior in a fashion that would raise the first player's fitness. The indirect approach has been developed extensively in the economics literature, along with various critiques. Our approach in terms of behavior would arise in the indirect approach if agents were not able to read a particular spouse's preferences, despite full knowledge of the distribution of preferences among the set of all potential spouses.

Why are Private Choices Distorted? - It is worth reiterating the source of the inefficiency here. Parents here make their private savings decisions simultaneously and non-cooperatively, and offspring are a public good, so it is not surprising that

is, if $T R R_{k}-T R R_{m}>0$, then $r_{k}-r_{m}>0$; furthermore $T R R_{k}-T R R_{m}$ underestimates $r_{k}-r_{m}$. That is, the actual difference between the private and public rates of return might exceed $1.18 \%$, and thus be closer to the quantitative prediction of the model.

${ }^{22}$ There is no shortage of candidates for such disasters-ice-ages being only the best-known. 
there is a free-rider problem. Indeed, there is undercontribution by each parent to the private capital that will generate income for each child. However, the issue is more subtle than this suggests. Undercontributing to the private capital of each child is achieved by producing too many children. What does too little investment in private capital imply? It limits the reproductive options of one's children. For simplicity, think of this as reducing the number of grandchildren. What is the basic reason for the distortion then? Although the effect of sex is to dilute one's concern with children by a factor of $1 / 2$, the dilution of concern with grandchildren is even greater, involving a factor of $1 / 4$. Hence sex creates a distortion from an attempt to favor the quantity of children over the quantity of grandchildren.

Cooperation between Parents - As a general biological phenomenon, it is not surprising that mating systems might be inefficient. In the vast majority of mammals, for example, males make no investment at all in their offspring beyond the purely genetic. Under plausible conditions of concavity of the offspring production function, this will be inefficient from the perspective of the couple. In the present model, we consider only symmetric equilibria, but this does not guarantee efficiency.

If the parents engage in repeated interactions, in contrast to the one-shot model here, there are familiar game-theoretic mechanisms that would reduce this inefficiency. Although this issue is sufficiently important and complex to merit further research, we content ourselves with some preliminary observations here. The key factor that limits efficiency in repeated interactions is divorce. Suppose parents are pair-bonded, but there is still a positive probability of splitting up, in each period. If the probability of splitting up is low, an outcome that is close to being fully efficient could be supported. ${ }^{23}$ If the probability of splitting up is high, on the other hand, only inefficient outcomes close to that described here, with an ESS choice of private capital, would be supported. ${ }^{24}$ Although repetition means that the present analysis would overstate the magnitude of the effect, divorce means that there would still be a tendency for the effective private rate of time preference to be too low. ${ }^{25}$

Global Warming - Finally, reconsider how this analysis might apply to the current debate about global warming. How might the current scenario concerning hunter-gatherers be translated to modern circumstances? We hypothesize that the private rate of time preference was, at least to some extent, hard-wired by evolution. We also hypothesize that public rates of time preference were similarly

\footnotetext{
${ }^{23}$ Indeed, this could be done with the threat of reversion to the ESS outcome.

${ }^{24}$ Even the threat of being "minimaxed" would then provide only a weak incentive to play in a more cooperative fashion.

${ }^{25} \mathrm{An}$ interesting possibility is that such game theoretic mechanisms could work without modifying preferences. The very success of these mechanisms would take the evolutionary pressure off lowering private rates of time preference. Hence a large underlying gap between private and public rates of time preference might reappear in novel circumstances.
} 
hard-wired, but at a lower level. ${ }^{26}$ That is, individuals evolved to desire that public decisions be made on the basis of a lower rate of time preference than the rate used in private decisions. ${ }^{27}$ Given the evolutionary basis of the public rate of time preference in maximizing population growth, the analysis might most convincingly be interpreted as a positive theory of public decisions, rather than an intrinsically ethical one. It predicts that public decisions about global warming would be made using a lower rate of time preference than used in private decisions, without having to be accepted as being the philosophical basis for such a lower rate. ${ }^{28}$ Indeed, we hypothesize that the link between fertility and population growth, on the one hand, and the rates of time preferences used in public and private decisions, on the other, has been severed..$^{29}$

Finally, we do not claim that the issue of global warming is represented precisely by our model. However, the model does capture, in a stylized form, intertemporal trade-offs that would have faced our hunter-gatherer ancestors. Then individuals could well apply the preferences derived in the model to decision problems which are different in technological detail.

\footnotetext{
${ }^{26}$ It is plausible that rates of time preference are also partly subject to enculturation, but this leads us still further from conventional economics.

${ }^{27}$ Individuals may vote for public choices from a less-than-perfectly-selfish perspective. Feddersen and Sandroni (2006) present a model in which voters are motivated by ethical obligation. We extend this notion to time preference.

${ }^{28}$ See Binmore (2005) for an argument that we should take evolution seriously as the foundation of our expressed ethical sense.

${ }^{29}$ Key here is the advent of modern birth control.
} 


\section{ApPENDix-Proofs}

LEMMA 1: For all $m, k \geq 0$, the equation (3) has a unique solution $c(m, k) \geq 0$. Further, $c(m, k)$ is continuously twice differentiable.

\section{PROOF OF LEMMA 1:}

If $m=0$ or $k=0$ then the only solution is $c=0$. If $m, k>0$ then $(m+k+$ c) $f(c, c)$ is less than $g(m, k)$ at $c=0$, greater than $g(m, k)$ if $c$ is large enough, and strictly increasing in $c$. Hence there exists a unique solution $c(m, k)>0$, which is clearly twice continuously differentiable.

\section{PROOF OF THEOREM 1:}

The solution cannot involve $m=0$ or $k=0$ since this implies $c=0$ and $c>0$ is feasible. Further, since $g_{m}(m, k) \rightarrow 0$ as $k \rightarrow \infty$, it follows that $g(m, k) /(m+$ $k+c) \rightarrow 0$ as $k \rightarrow \infty$, so that $c \rightarrow 0$. Similarly, $c \rightarrow 0$ as $m \rightarrow \infty$. Hence there must exist an interior optimum with $m, k>0$. Hence $c_{m}(m, k)=c_{k}(m, k)=0$. It then follows from differentiating $(m+k+c) f(c, c)=g(m, k)$ that $g_{m}(m, k)=$ $g_{k}(m, k)=f(c, c)$.

Further, there is only one solution to these first order conditions. It is straightforward to show that, for each $m>0$ there exists a unique $k(m)$ such that $g_{m}(m, k(m))=g_{k}(m, k(m))$. Further, $k(m) \rightarrow 0$ as $m \rightarrow 0$ and $k(m)$ is continuously twice differentiable. Define $\hat{c}(m)=c(m, k(m))$. Upon differentiating the constraint twice and then setting $c_{m}(m, k)=c_{k}(m, k)=0$, we obtain, dropping most arguments for simplicity,

$$
\left(1+k^{\prime \prime}+\hat{c}^{\prime \prime}\right) f(\hat{c}, \hat{c})+(m+k+\hat{c})\left(f_{1}(\hat{c}, \hat{c})+f_{2}(\hat{c}, \hat{c})\right) \hat{c}^{\prime \prime}=g_{m m}+2 g_{m k} k^{\prime}+g_{k k}\left(k^{\prime}\right)^{2}+g_{k} k^{\prime \prime} .
$$

Since $g$ is strictly concave, $g_{m m}+2 g_{m k} k^{\prime}+g_{k k}\left(k^{\prime}\right)^{2}<0$. Recalling that $g_{k}=f(c, c)$, it follows that $\hat{c}^{\prime \prime}<0$ at any solution of the first order conditions. But this implies there can only be one such solution, so there is a unique optimum as well.

LEMMA 2: For given $m, \bar{k}, \bar{c}>0$, the constraint

$$
2(m+k+c) f(\bar{c}, c)=g(m, \bar{k})+g(m, k)
$$

may have no solution for $c$ for some $k \geq 0$. However, the set of feasible $k$ 's is a non-degenerate interval. On this interval, there is a unique solution $c(k)$ which is twice differentiable. ${ }^{30}$ Further, given each $m>0$, there is a unique (feasible) best reply, $c(\bar{c})$, say, for the mutant to each $\bar{c}>0$. This is also the only solution to the first order condition

$$
2 f(\bar{c}, c)=g_{k}(m, k) .
$$

${ }^{30}$ We suppress, for the moment only, that $c$ is also a function of $m$. 


\section{PROOF OF LEMMA 2:}

The constraint $2(m+k+c) f(\bar{c}, c)=g(m, \bar{k})+g(m, k)$ need not be capable of being satisfied for all $k \geq 0$. However, it always satisfied with $k=\bar{k}>0$ and $c=\bar{c}>0$. Over the domain where it can be satisfied, there is a unique $c(k)$, for each $k$. The function $c(k)$ is strictly quasiconcave, and twice continuously differentiable. At the edges of this domain either $k=0$ or $c=0$. It follows that the domain is a non-degenerate interval. If $k=0$ is in the domain, it cannot be optimal since then $c^{\prime}(0) \leq 0$ but $g_{k}(m, k) \rightarrow \infty$ as $k \rightarrow 0$. It follows that there is an interior best reply. Differentiating the constraint once and setting $c^{\prime}(k)=0$ yields that $2 f(\bar{c}, c)=g_{k}(m, k)$. Differentiating the constraint twice and then setting $c^{\prime}(k)=0$ yields

$$
2 c^{\prime \prime}(k) f(\bar{c}, c)+2(m+k+c) f_{2}(\bar{c}, c) c^{\prime \prime}(k)=g_{k k}(m, k) \text { so } c^{\prime \prime}(k)<0 .
$$

It follows that there can only be one such solution of $c^{\prime}(k)=0$. Hence there is a unique best reply, $c(\bar{c})$, as well.

\section{PROOF OF THEOREM 2:}

Consider the conditions characterizing the mutant best reply $2 f(\bar{c}, c)=g_{k}(m, k)$ and $2(m+k+c) f(\bar{c}, c)=g(m, \bar{k})+g(m, k)$. If $\bar{c}=0$ then $2 f(0, c)=g_{k}(m, k)>$ 0 so $c>0$. On the other hand, if $\bar{c}$ is large enough, the conditions cannot be satisfied. At the largest feasible $\bar{c}, c=0$. Over the feasible domain, let $c(\bar{c})$ and $k(\bar{c})$ denote the solutions for $c$ and $k$. Now differentiating the second condition yields $2\left(k^{\prime}(\bar{c})+c^{\prime}(\bar{c})\right) f(\bar{c}, c)+2(m+k+c)\left(f_{1}(\bar{c}, c)+f_{2}(\bar{c}, c) c^{\prime}(\bar{c})\right)=g_{k} k^{\prime}(\bar{c})$. Using the first condition, it follows that $c^{\prime}(\bar{c})<0$. Hence there exists a unique $\bar{c}$ such that $c(\bar{c})=\bar{c}>0$. This is then an ESS because $c=\bar{c}$ is the unique best reply to $\bar{c}$. Indeed, from the above construction, this is is the only ESS in pure strategies.

\section{PROOF OF THEOREM 3:}

If $m=0$, then $c=0$, which is not optimal since $m>0$ implies $c>0$. If $m \rightarrow \infty$, then $f(c, c)=g(m, k) /(m+k+c) \rightarrow 0$, since $g_{m}(m, k) \rightarrow 0$. Hence $c \rightarrow 0$ as $m \rightarrow \infty$. It follows that there exists an interior solution. From Theorem 2 , the constraints

$$
(m+\bar{k}+\bar{c}) f(\bar{c}, \bar{c})=g(m, \bar{k}) \text { and } g_{k}(m, \bar{k})=2 f(\bar{c}, \bar{c})
$$

determine $\bar{k}$ and $\bar{c}$ as functions of $m$. Let these functions be $\bar{k}(m)$ and $\bar{c}(m)$, say, which are continuously differentiable. At any interior solution, $\bar{c}^{\prime}(m)=0$ so that, from the second constraint-

$$
g_{k m}(m, \bar{k})+g_{k k}(m, \bar{k}) \bar{k}^{\prime}(m)=0 \text { so that } \bar{k}^{\prime}(m)=-\frac{g_{k m}(m, \bar{k})}{g_{k k}(m, \bar{k})} \geq 0,
$$


since $g_{k m}(m, \bar{k}) \geq 0$ and $g_{k k}(m, \bar{k})<0$. The first constraint then yields

$$
f(\bar{c}, \bar{c})\left(1+\bar{k}^{\prime}(m)\right)=g_{m}(m, \bar{k})+g_{k}(m, \bar{k}) \bar{k}^{\prime}(m) .
$$

Using $g_{k}(m, \bar{k})=2 f(\bar{c}, \bar{c})$ again, it follows that, at the constrained best choice of $m=\bar{m}$,

$$
g_{m}(\bar{m}, \bar{k}(\bar{m}))=f(\bar{c}(\bar{m}), \bar{c}(\bar{m}))\left(1-\bar{k}^{\prime}(\bar{m})\right) \leq f(\bar{c}(\bar{m}), \bar{c}(\bar{m})) .
$$




\section{REFERENCES}

Afonzo, Antonio, and Miguel St Aubyn. 2010. "Public and Private Investment Rates of Return: Evidence for Industrialized Countries." Applied Economics Letters, 17(9): 839-843.

Alesina, Alberto, and Dani Rodrik. 1994. "Distributive Politics and Economic Growth." Quarterly Journal of Economics, 109(2): 465-490.

Bergstrom, Theodore C. 1995. "On the Evolution of Altruistic Ethical Rules for Siblings." American Economic Review, 85(1): 58-81.

Binmore, Ken. 2005. Natural Justice. Oxford:Oxford University Press.

Boyd, Robert, and Peter J. Richerson. 1990. "Group Selection Among Alternative Evolutionarily Stable Strategies." Journal of Theoretical Biology, 145(3): 331-342.

Feddersen, Timothy, and Alvaro Sandroni. 2006. "A Theory of Participation in Elections." American Economic Review, 96(4): 1271-1282.

Hansson, Ingemar, and Charles Stuart. 1990. "Malthusian Selection of Preferences." American Economic Review, 80(3): 529-44.

Nordhaus, William. 1994. Managing the Global Commons: The Economics of Climate Change. Cambridge, MA:MIT Press.

Nordhaus, William. 2007. "The Stern Review on the Economics of Climate Change." Journal of Economic Literature, 45(3): 686-702.

Pigou, Arthur C. 1912. Wealth and Welfare. London:MacMillan and Co.

Ramsey, Frank P. 1928. "A Mathematical Theory of Saving." The Economic Journal, 38(152): 543-559.

Robson, Arthur J., and Balázs Szentes. 2008. "Evolution of Time Preference by Natural Selection: Comment." American Economic Review, 98(3): 11781188 .

Robson, Arthur J., and Larry Samuelson. 2007. "The Evolution of Intertemporal Preferences." American Economic Review P\&P P, 97(2): 496-500.

Robson, Arthur J., and Larry Samuelson. 2009. "The Evolution of Time Preference with Aggregate Uncertainty." American Economic Review, 99(5): 1925-53.

Robson, Arthur J., and Larry Samuelson. 2010. "The Evolutionary Foundations of Preferences." In Handbook of Social Economics. , ed. Alberto Bisin and Matt Jackson, 221-310. North-Holland. 
Robson, Arthur J., and Myrna Wooders. 1997. "On the GrowthMaximizing Distribution of Income." International Economic Review, 38(3): 511-26.

Robson, Arthur J., Balázs Szentes, and Emil Iantchev. 2012. "The Evolutionary Basis of Time Preference: Intergenerational Transfers and Sex." American Economic Journal: Microeconomics, 4(4): 172-201.

Rogers, Alan R. 1994. "Evolution of Time Preference by Natural Selection." American Economic Review, 84(2): 460-481.

Sidgwick, Henry. 1874. The Methods of Ethics. Oxford:Oxford University Press.

Smith, John Maynard. 1964. "Group Selection and Kin Selection." Nature, 201(4924): 1145-1147.

Stern, Nicholas. 2006. Stern Review on the Economics of Climate Change. London:Her Majesty's Treasury.

Williams, George C. 1972. Adaptation and Natural Selection: A Critique of Some Current Evolutionary Thought. Princeton:Princeton University Press. 